\title{
A Model for Scale-Degree Reinterpretation: Melodic Structure, Modulation, and Cadence Choice in the Chorale Harmonizations of J. S. Bach
}

\author{
TREVOR de CLERCQ[1] \\ Middle Tennessee State University
}

\begin{abstract}
This paper reports a corpus study of the 371 chorale harmonizations by J. S. Bach. Specifically, this study investigates what kinds of events are typical at phrase endings given various melodic conditions, i.e., how well melodic structure is a predictor of modulation and cadence choices. Each fermata event was analyzed by ear and encoded with regard to the local key area and the cadence type. The frequency of each cadence type was then tabulated with respect to categorizations of the melodic structure (in terms of the intervallic pattern and scale degree content) prior to and including the cadential arrival. It is shown that most fermata events can be categorized by a small collection of event types. As a result, a simplified conceptual model of cadence choice is posited. This model proposes that a basic harmonization default is to (re-)interpret the soprano note at the fermata as scale-degree 1, 2, or 3 in some closelyrelated key area via an authentic or half cadence. The efficacy of this model is found to be very good, especially given a stepwise melodic descent into the fermata. Moreover, an overall success rate above $90 \%$ can be achieved by accounting for other cadence types through the specific musical contexts in which they occur.
\end{abstract}

Submitted 2014 May 29; accepted 2015 April 9.

KEYWORDS: J. S. Bach, chorale harmonization, corpus study, cadences, pedagogy

IN a 2009 article, Robert Gauldin describes his experience developing and teaching a styles simulation course for graduate music theory students at the Eastman School of Music, during which much time is devoted to Baroque chorale harmonization in the style of J. S. Bach. The Bach chorale is a particularly appropriate topic for a styles simulation course targeted at graduate music theory students, most of whom will go on to teach music theory at the college level, since four-part chorale harmonization is a common activity within the undergraduate music theory classroom (or at least, four-part chorale harmonization is a common activity within American undergraduate music theory textbooks, e.g., Kostka, Payne, \& Almén, 2013; Clendinning \& Marvin, 2010; Laitz, 2012). We may guess (or at least hope) that graduate-level music theory students would be quite good at this task already, given the extent to which we presume their undergraduate education covered this topic.

But while Gauldin notes that the part-writing ability of his graduate students is typically adequate, he finds that other areas are in need of repair. One of his main laments is that students - when harmonizing a hymn melody in the style of Bach - often have a kind of "tunnel vision" that causes them to interpret melodic fragments within only a single tonal center; moreover, this tunnel vision inhibits students from reinterpreting these melodic fragments in key centers other than the global tonic, such that student harmonizations tend to lack the tonal variety of a Bach setting. In short, students tend to avoid cadences that involve modulation, resulting in harmonizations not representative of the Bach chorale style.

As an example of this issue, consider the opening bars of the chorale melody "Wie schön leuchtet der Morgenstern" shown in Figure 1. An approach suffering from tunnel vision would harmonize all three of these phrases within the tonic key (here, F major). Indeed, interpreting these three phrases solely within the realm of tonic is a valid harmonic and contrapuntal possibility. For instance, we might imagine that the first phrase could end with a half cadence in tonic, as shown in the hypothetical four-part harmonization of Figure 2.[2] (The reader can imagine similar harmonization strategies for the two phrases that follow.) 


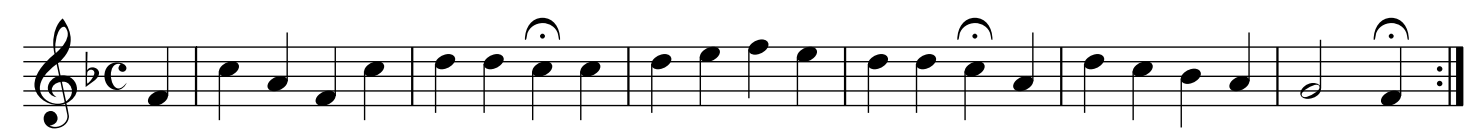

Fig. 1: Opening bars of the hymn melody "Wie schön leuchtet der Morgenstern."

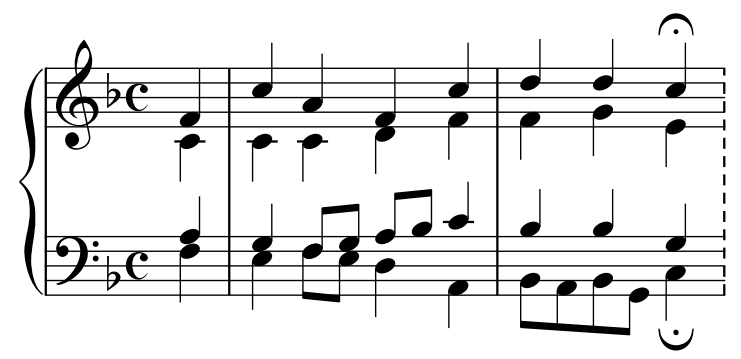

Fig. 2. Hypothetical harmonization of the first phrase of "Wie schön leuchtet der Morgenstern" with a half cadence in tonic.

To combat this tonic-key tunnel vision, Gauldin introduces the concept of "scale-degree reinterpretation," a term that describes the process of reinterpreting the notes of the hymn melody as diatonic scale degrees in some key other than tonic. Gauldin offers a few illustrations of this technique, as shown in Figure 3. For instance, given a chorale phrase that ends with the notes D-D-C in the global key of F major (such as the first phrase in Figure 1), we could reinterpret the melody as scale-degrees 4-4-3 in the local key of A minor via an imperfect authentic cadence (bar 3 in Figure 3); or we might reinterpret the melody as scale-degrees 3-3-2 in the local key of Bb major via a half cadence (Figure 3, bar 4).

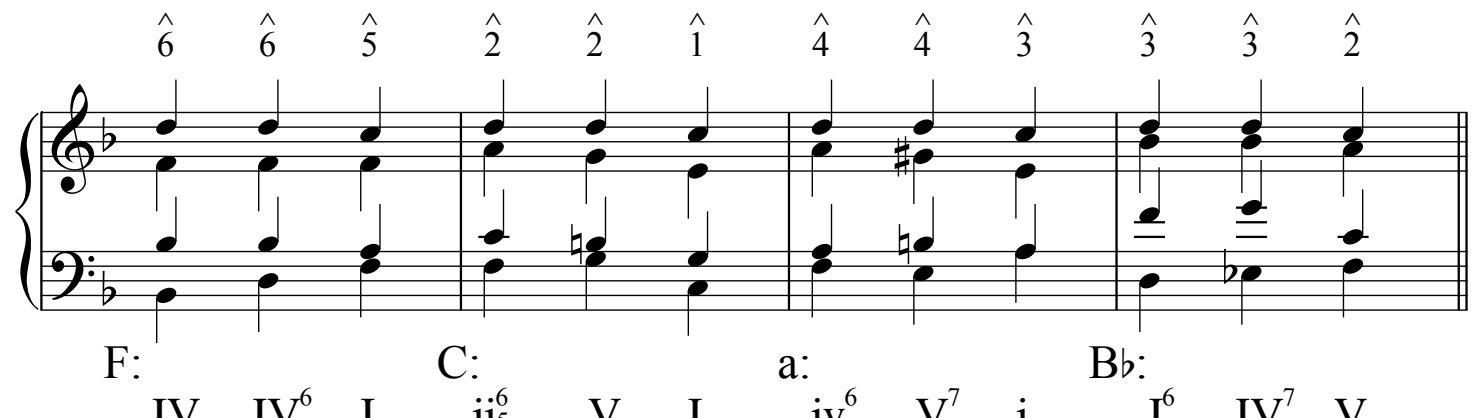

Fig. 3. Examples of scale-degree reinterpretation at cadences (adapted from Gauldin 2009)

But while scale-degree reinterpretation is, at least according to Gauldin, an important concept for simulating the Bach style of chorale harmonization, it does come at a cost. Tonic-key tunnel vision has a distinct advantage, in that it creates a small and relatively manageable problem space for the student weighing cadence options. For instance, if a melodic phrase ends on scale-degree 5, a student with tonickey tunnel vision faces only three cadential possibilities (assuming typical American undergraduate textbook cadence categories): a half cadence in tonic, a plagal cadence in tonic, or an imperfect authentic cadence in tonic. With scale-degree reinterpretation, the cadential possibilities increase significantly. For example, there are at least ten cadence possibilities (again, assuming typical American undergraduate textbook cadence categories) given the melodic phrase ending D-D-C in an F major chorale, as shown in Table 1. Admittedly, ten is not an inordinately large number, but one should realize that each of these ten generic cadence categories encompasses a great variety of specific realizations. The large number of realworld possibilities can be especially overwhelming if one is trying to harmonize a chorale by sight at the keyboard, a common task in graduate music theory training so as to prepare future teachers for impromptu classroom demonstrations.

It would thus be nice to narrow down the choices involved with scale-degree reinterpretation to those that are most typical. This winnowing of options requires knowledge about the interaction between melodic structure, key area, and cadence choice. We might ask: How well does a particular melodic 
structure predict the cadence and local key area within the chorale harmonizations of Bach? When is scaledegree reinterpretation stylistic, and when is it not? Are certain types of scale-degree reinterpretation more typical than others? These sorts of questions remain unanswered in Gauldin's 2009 article.

Table 1. Local cadence possibilities for a melodic phrase ending with global scale-degrees 6-6-5

\begin{tabular}{|c|c|c|c|}
\hline Local scale-degrees & Local Key & Cadence Type & Sample Harmonization \\
\hline $6-6-5$ & I & Plagal & $I V-I V^{6}-I$ \\
\hline $6-6-5$ & I & Half & IV $-\mathrm{ii}^{6}-\mathrm{V}$ \\
\hline $6-6-5$ & I & Imperfect Authentic & $I V^{6}-v^{2 i i^{7}}-I$ \\
\hline $2-2-1$ & $\mathrm{~V}$ & Perfect Authentic & $\mathrm{ii}^{6}-\mathrm{V}-\mathrm{I}$ \\
\hline $2-2-1$ & $\mathrm{~V}$ & Deceptive & $\mathrm{ii}^{6}-\mathrm{V}-\mathrm{vi}$ \\
\hline $2-2-1$ & $\mathrm{~V}$ & Imperfect Authentic & ii $-V^{6}-I$ \\
\hline $4-4-3$ & iii & Imperfect Authentic & $i v^{6}-V^{7}-i$ \\
\hline $4-4-3$ & iii & Deceptive & $i v^{6}-V^{7}-V I$ \\
\hline $4-4-3$ & iii & Plagal & $i v-i v^{6}-i$ \\
\hline $3-3-2$ & IV & Half & $I^{6}-I V^{7}-V$ \\
\hline
\end{tabular}

Of course, musicologists have been giving empirically-informed advice on the typical cadence choices in the Bach chorales since at least the statistical work of McHose (1947). But although typical cadence types are discussed, the interaction between melodic structure, key area, and cadence remains underexplained. Consider, for example, the chart (reproduced in Table 2) found in Boyd's book on the Bach chorale (1967/1999). While this table contains interesting information on the frequencies of different cadence types, the lack of finer detail compromises its ability to convey much practical knowledge. In particular, it is not clear from Boyd's table (or his accompanying text) why or in what context (melodic or otherwise) to use each cadence type. This issue is also prevalent in modern undergraduate theory textbooks (like those mentioned earlier), where cadence types are presented as a list of available options without much discussion (if any) of the melodic and modulatory contexts in which these cadence types normally occur.

Table 2: Analysis of cadences in 371 Bach chorales, adapted from Boyd (1967/1999).

\begin{tabular}{ll|lll|l}
\hline Cadence & $\begin{array}{l}\text { Harmonic } \\
\text { Motion }\end{array}$ & $\begin{array}{l}\text { Root } \\
\text { Position }\end{array}$ & Inverted & Total & $\begin{array}{l}\text { Approx. } \\
\text { Percent }\end{array}$ \\
\hline Authentic & V - I & 1,241 & 211 & 1,452 & 73.0 \\
Half & $?-$ V & 225 & 190 & 415 & 21.0 \\
Plagal & IV - I & 30 & 14 & 44 & 2.0 \\
Deceptive & V - VI & 33 & 0 & 33 & 1.5 \\
Others & n/a & n/a & n/a & 50 & 2.5 \\
\hline
\end{tabular}

Perhaps the best pedagogical information on chorale cadences can be found in Gauldin's own textbook on Baroque counterpoint (1988/1995). In his discussion of the chorale, Gauldin offers a collection of typical cadential formulas, reproduced here as Figure 4. Of particular value is the presentation of each cadence not simply as a generic cadence label but rather as an outer-voice counterpoint, such that the student has a good idea about not only the typical harmonic motions of the cadence types but also the melodic structures these harmonic motions will usually support. Nonetheless, it remains unclear how to handle melodic endings that do not conform to this handful of cadential patterns. For instance, what cadence(s) would be most typical given a melody that descends to scale degree 5 by step at the end of a phrase (such as the first phrase in Figure 1)? What about melodic phrases ending on scale degree 4 or 6 ? And how does this collection of mostly major-key cadences map to minor keys? In addition, note that none of the cadences in Figure 4 leave the tonic key. Gauldin does touch on the topic of modulation in his text, but he does not discuss which melodic factors tend to engender it. In other words, he does not connect these typical cadences with his concept of scale-degree reinterpretation. 


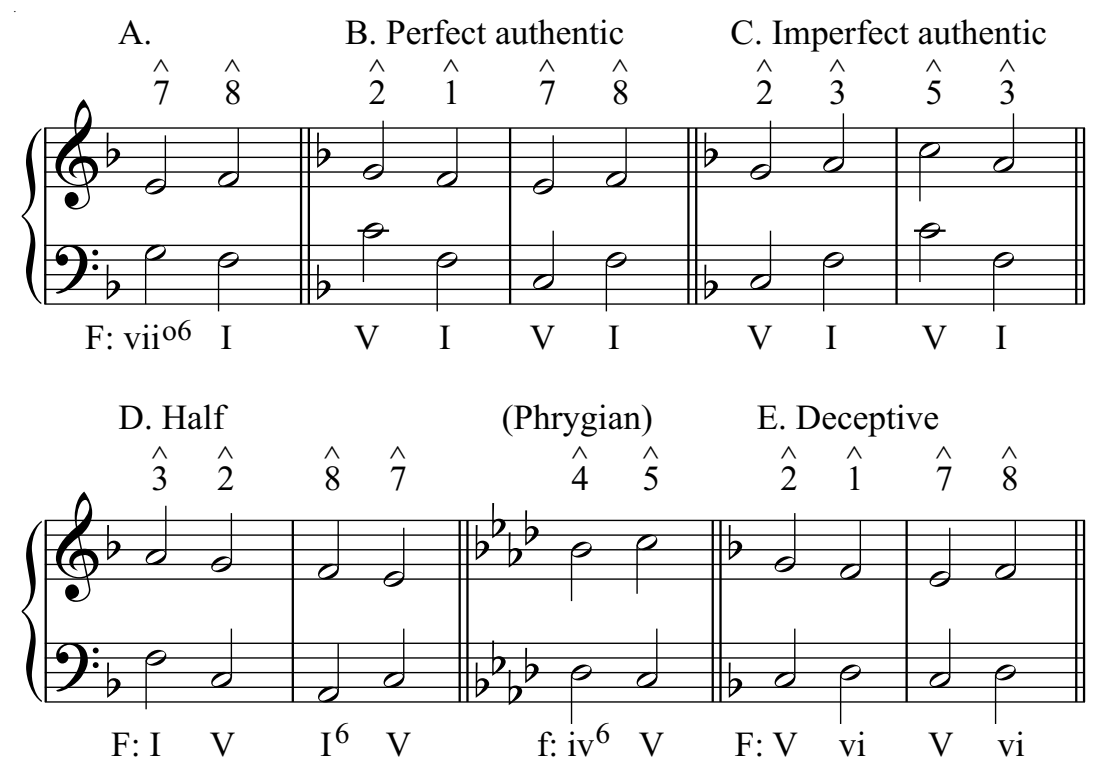

Fig. 4. Typical cadential formulas from Gauldin 1988/1995 (p. 44).

In order to answer these sorts of questions about the relationship between melody, modulation, and cadence types in the Bach chorales, further empirical research seems appropriate. In particular, a statistical corpus study is well suited to compare the melodic structure at phrase endings to the frequency of various cadence types and modulation levels. Historically speaking, corpus work with the chorale harmonizations of Bach has been a popular research area, in part due to the relatively limited complexity of the chorales in terms of texture and rhythm. Aside from those mentioned earlier, corpus studies with the Bach chorales have not sought to explain the specific task of chorale harmonization; rather, their scopes have been fairly broad. Rohrmeier and Cross (2008), for example, have used the chorales to investigate fundamental aspects of tonality and harmonic syntax, showing that only a few elements control most of the musical structure. We also find wide-ranging aims in Tymoczko (2011), as he combines the Bach chorales with a corpus of Mozart piano sonatas to support a descending-thirds model of tonal harmony. Recent articles by Quinn and Mavromatis address other large-scale issues via corpus work with the chorales, from postulating algorithms for key-finding (2010) to investigating the extent by which voice-leading implies harmonic function (2011).

Although this prior corpus work has not focused on recreating the chorale style of Bach, it should be mentioned that a number of researchers have attempted to recreate the Bach chorale through automated music composition projects. These projects focus on getting a computer, rather than a human student, to create a convincing chorale harmonization. A few different approaches have been used in this regard, including constraint-based methods, in which the automated harmonization task relies on a knowledge base drawn from theory treatises (Ebcioğlu, 1988, 1990); neural networks, where the program is trained via its own analyses (Hörnel \& Menzel, 1998); and Markov chains, in which transitions between different states are determined via probabilistic methods (Thorpe, 1998; Biyikoglu, 2003; Allan \& Williams, 2005). Some of these models have been fairly successful at creating convincingly stylistic harmonizations. Unfortunately, these studies have limited benefits for music theory pedagogy; even when these models are successful, it is difficult to infer any practical advice to a music student since a wide variety of parameters and settings are involved.

In the corpus study reported here, the parameters are purposefully limited so as to increase the pedagogical applicability. Specifically, this study investigates what kinds of modulatory and cadential events are typical at phrase endings given the various melodic structures of the 371 chorale harmonizations by J. S. Bach. With this information, we can better know when and to where it is advisable to modulate at phrase endings if we want to simulate the Bach chorale style, i.e., how to best apply the scale-degree reinterpretation method that Gauldin describes. It will be shown that most events at phrase endings can be categorized via a small collection of specific cadence types. As a result, we can posit a simplified conceptual model of cadence choice, meant to benefit both music theory teachers and students. The 
efficacy of this model is shown to be very good, especially given certain conditions, requiring only a few additional concepts to achieve a high rate of success.

\section{METHODS AND METHODOLOGICAL ISSUES}

The first concern for this study was the corpus itself. A number of different editions of the Bach chorales have been published in the roughly 250 years since "Breitkopf und Sohn" offered the first known collection for sale.[3] Because the chorales were not composed as a single standalone work but instead excerpted from a variety of much longer works - such as cantatas, oratorios, and passions (many of which have since been lost) - it would be difficult to claim that a definitive edition exists. For this study, the 1941 edition by Riemenschneider was used (republished in 1986), if only because of its popularity.[4] All references to a particular chorale will be via its Riemenschneider number. (For example, "Aus meines Herzens Grunde," BWV 269, will be referred to as chorale 1.)

Although the corpus contains 371 chorale harmonizations, the total number of unique harmonizations that can be attributed to Bach is somewhat smaller. In one case, the harmonization is by another composer.[5] In other cases, we find pairs of chorales with identical harmonizations, despite differences in the keys or names of the chorales. All told, there exist 24 duplicate harmonizations in the collection.[6] The final corpus reported here thus consists of only 346 chorales. For the sake of convenience, the lower-numbered chorale of any identical pair is included in the corpus and the highernumbered "copy" is ignored.[7]
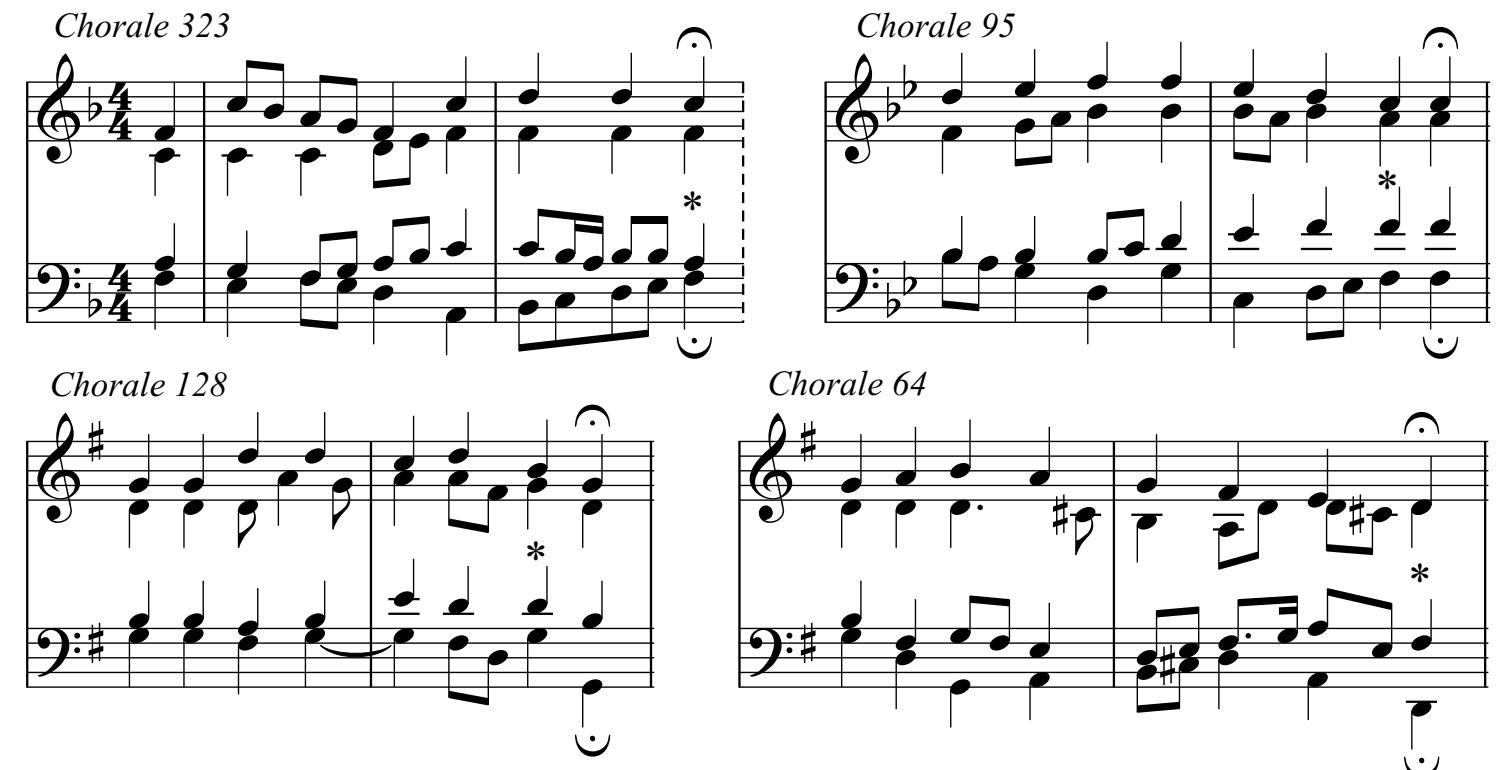

Chorale 64

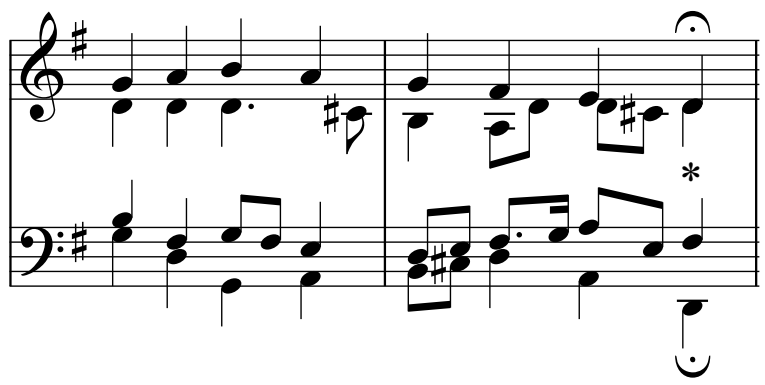

Fig. 5. Different cadential arrivals $(*)$ in the opening bars of four Bach chorales.

The next step was to determine the location of each phrase and phrase ending. As a rule, fermatas were taken to delineate phrases but not necessarily to indicate the exact location of the cadential arrival. Figure 5 shows four different scenarios. Most commonly, the final chord of the cadence occurs under the fermata on beat 3 (as in chorale 323). But cadential arrivals and fermatas do not always align. The exceptions typically occur when the fermata appears on a weak beat (i.e., beats 2 and 4 in 4/4; beats 2 and 3 in 3/4). In these situations, Bach exhibits a clear tendency to shift the cadential arrival to the strong beat when possible. If the weak-beat fermata is preceded by a unison (chorale 95) or a fall of a third (chorale 128), the cadential arrival will normally occur on the strong beat prior to the fermata. (This practice is possible since the intervals of a unison and third can be contained within the same harmony.) In the case of weak-beat fermatas that are preceded by a step (chorale 64), it is not possible to shift the cadential arrival to the preceding strong beat (assuming that a triad is the cadential goal), and so the cadential arrival is displaced forward from its typical strong-beat location to coincide with the weak-beat fermata. Overall, the preference for cadences on strong beats can be considered a general feature of the chorales, whether in $4 / 4$ 
or $3 / 4$ meter. In this corpus study, cadence locations were determined on a case-by-case basis, with the cadential arrival (if any) taken as the last change of harmony at or before the fermata.

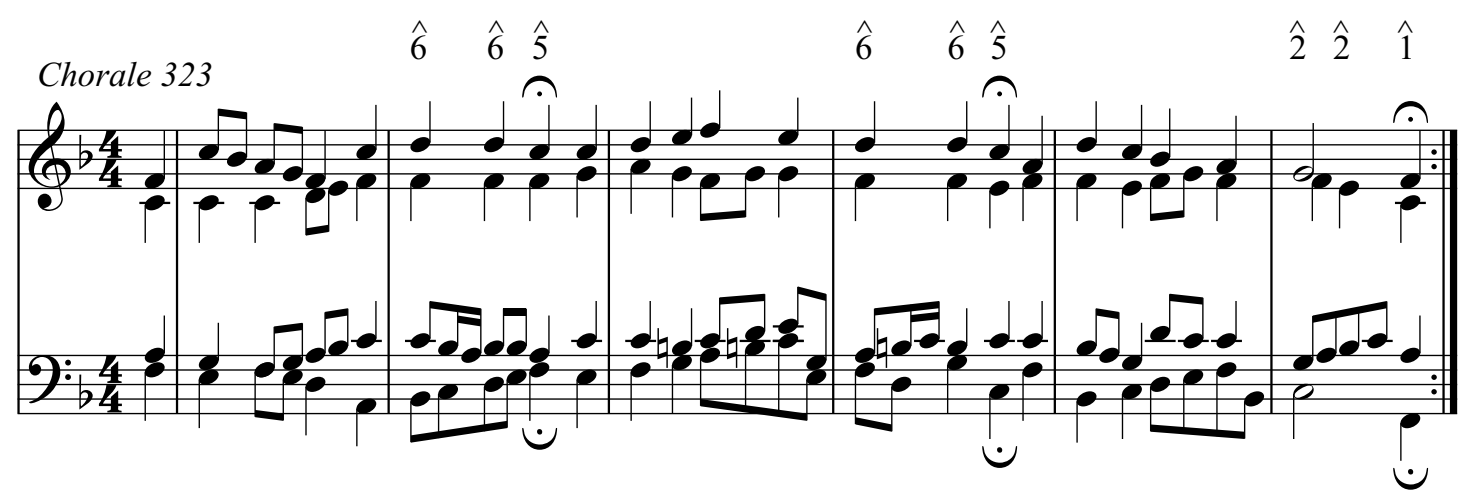

Fig. 6. Melodic scale-degree encodings for chorale 323 (first six bars).

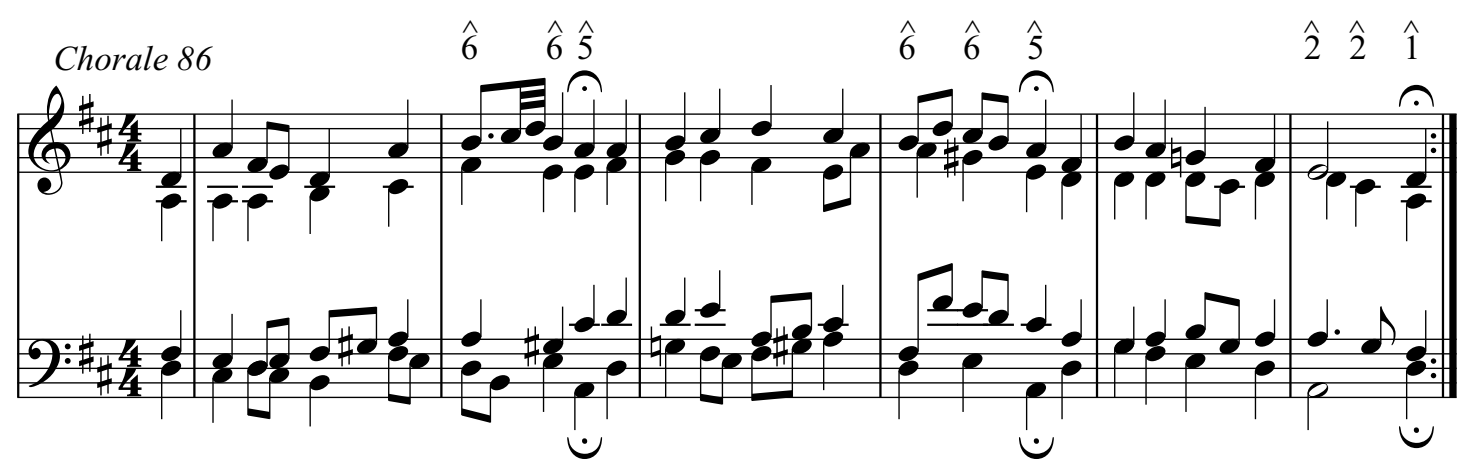

Fig. 7. Melodic scale-degree encodings for chorale 86 (first six bars).

With cadence locations identified, the scale-degree content of the melody was encoded in terms of the global tonic for the last three beats of the cadence. Figure 6 shows a sample encoding for the beginning of chorale 323. (Observe how the half note at the end of the excerpt is encoded as two instances of scaledegree 2 because it lasts two beats.) In some cases, non-harmonic tones had to be stripped away, which required some analytical decisions. Figure 7 shows the opening bars of chorale 86, whose surface melody differs from that of chorale 323 . Yet both chorales are based on the same underlying hymn melody and thus share the same melodic encoding. (These sorts of decisions were made on a case-by-case basis.) Finally, the melodic encodings were stored in a tab-delimited text file (see Figure 8).[8] To best handle the modal nature of some hymn melodies (more on that issue below), scale degrees were universally measured in terms of a parallel major scale, with flats and sharps indicating raised or lowered versions (e.g., scaledegree 3 in a minor key was encoded as "b3").

$\begin{array}{lllllll}1 & 2.3 .2 . & 2.2 .1 . & 4.3 .2 . & 3.3 .1 . & 2.3 .2 . & 2.2 .1 . \\ 2 & \text { b76.5. } & 7.6 .5 . & 2.3 .2 . & 2 . \# 12 . & 4.3 .2 . & 3.2 .1 . \\ 3 & 5.632 . & \text { b32.1. } & \text { b71.2. } & 1.7 .1 . & 4 . \mathrm{b} 32 . & \\ 4 & 6.5 .4 . & 5.6 .5 . & 1.6 .5 . & 5.4 .3 . & 6.7 .1 . & \\ 5 & 4.4 .3 . & 2.2 .1 . & 3.2 .1 . & 3 . \# 45 . & 4.4 .3 . & \text { (etc.) } \\ 6 & 5.5 .3 . & 3.2 .3 . & 6.6 .5 . & 2.2 .1 . & & \\ 7 & 2.2 .3 . & 2.2 .1 . & 7.7 .6 . & 6.6 .5 . & 3.3 .1 . & \text { (etc.) } \\ 8 & 2.2 .1 . & 4.4 . \mathrm{b} 3 & 2.2 .1 . & 4.4 .5 . & 5.5 .4 . & \text { (etc.) } \\ 9 & 5 . \# 45 . & 2.2 .1 . & 3.3 .2 . & 6 . \# 56 . & 2.2 .2 . & 2.2 .1 . \\ 10 & \text { b71.2. } & \text { b7b65. } & 4.2 .1 . & 2.1 . b 7 & 5.5 .5 . & \end{array}$

Fig. 8. Excerpt of tab-delimited text file with melodic encodings. Column 1 lists the chorale number, and each successive column lists, for each fermata in the chorale, the scale-degree content of the melody for the three beats up to and including the cadence. (Dots are placeholders for scale degrees without accidentals.) 
After encoding the scale-degree content of the melody, the next step was to encode the local key areas and cadence types for phrase endings. To facilitate this process, I used a custom shorthand that tracks three aspects of a cadence: 1) the local key area, 2) the cadence classification, and 3) the chordal tone of the soprano at the cadential arrival. Local key areas are indicated with traditional upper and lower case Roman numerals (representing major and minor key areas, respectively) as reconciled with the global tonic. (For instance, ii in $\mathrm{C}$ major is a cadence in the local key of $\mathrm{D}$ minor.) The cadence classification is indicated with a two-letter abbreviation, which represents (with one exception noted below) the standard cadence categories found in American music theory textbooks: perfect authentic cadence (PA), imperfect authentic cadence (IA), half cadence (HF), phrygian cadence (PH), plagal cadence (PL), and deceptive cadence (DE). Finally, the chordal member of the soprano note is indicated with an Arabic number. Thus, the notation "III-IA3" in the key of G minor represents an imperfect authentic cadence in Bb major with the third of the chord (D) in the soprano at the point of cadential arrival. (I also indicated, via a slash [/], if the final chord of a cadential gesture was inverted, but this feature was rarely needed.) Figure 9 shows this notational system applied to the first two cadences of chorale 44 .

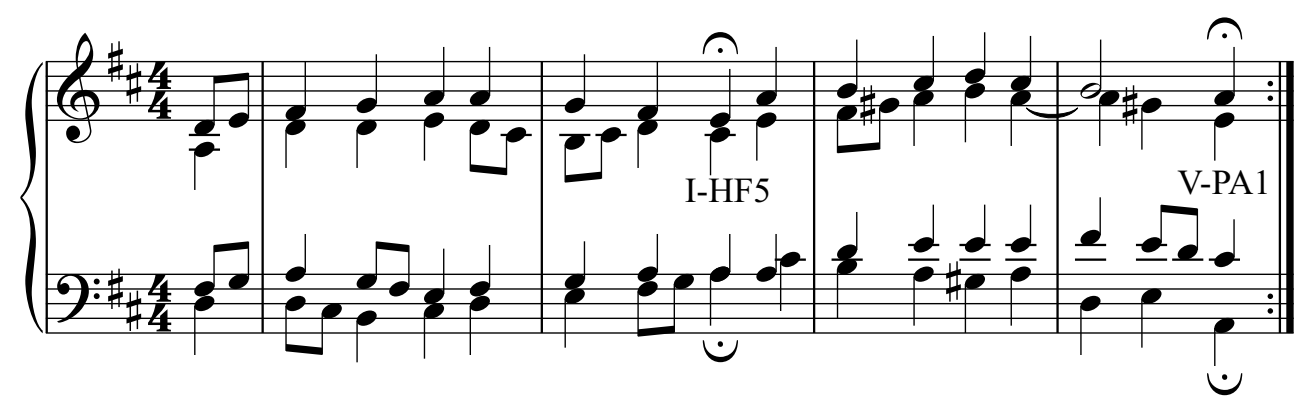

Fig. 9. Two cadences and their analyses from the beginning of chorale 44 .

In addition to the standard cadence classifications, I included one additional category that was discussed by McHose (1947) but has since fallen out of common parlance, even though (as we will see) this cadence type is common enough within the chorales to warrant its own category. This category is what McHose refers to as the "plagal half cadence," although I prefer to call it a "subdominant stop" (shorthand, "SS") to avoid confusion with other cadence types. An example of a subdominant stop can be found in bars 13-14 of chorale 1, as shown in Figure 10. Note how the F natural in bar 13, which tonicizes the C-major chord under the fermata, is not strong enough to make this arrival sound like an authentic cadence in the key of IV.

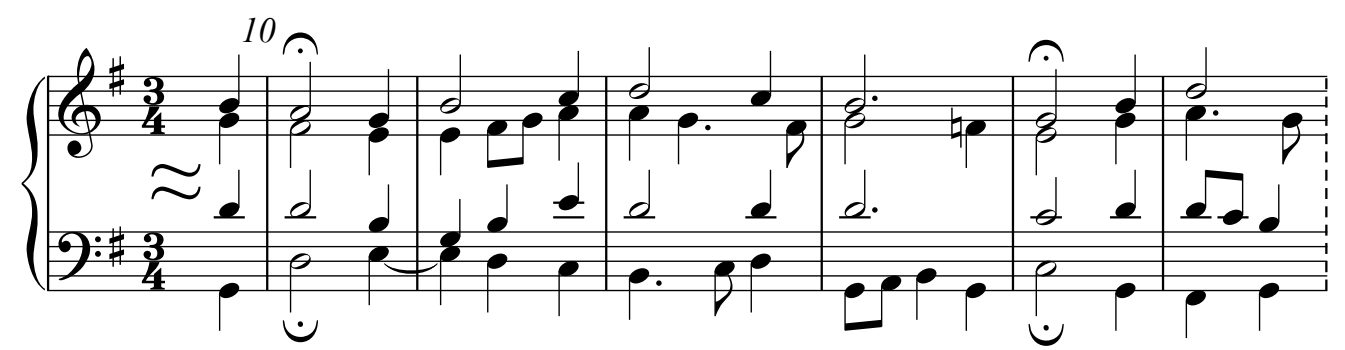

Fig. 10. A "subdominant stop" in chorale 1 (bars 13-14).

On a related topic, it may be worth clarifying what is meant by the word "cadence" itself. Some readers - especially those with Schenkerian leanings (see Caplin 2004) - may feel that the subdominant stop is not truly a cadence at all. This feeling may extend to the plagal cadence or the deceptive cadence as well. With regard to the current study, however, this issue is somewhat moot, since this project seeks to identify what types of harmonizations are typically engendered by a particular melodic phrase ending as indicated by a fermata. Accordingly, we could say that we seek knowledge about "fermata events," some of which may be true cadences in the Schenkerian sense, some of which may be not. In this paper, I will use the term "cadence" to mean "the harmonic event at the phrase ending as indicated by the fermata," if only because the former is less clumsy. 
The actual task of categorizing cadences was done by ear at the piano, using the shorthand described above, with the results stored in a separate tab-delimited text file (see Figure 11). This analytical task was not always a straightforward process. One issue is that some fermatas are not preceded by an event that warrants one of the cadence categories described above. Consider, for example, the excerpt from chorale 65 shown in Figure 12. Although the chord under the second fermata (E minor) deserves a submediant label, it does not sound like a deceptive cadence since the chord prior (B minor) does not generate any significant expectation for the major-key tonic. No other cadence classification seems appropriate, and so problematic cases like this were categorized simply as "no cadence" ("NC").

$\begin{array}{lllllll}1 & \text { I-HF5 } & \text { I-PA1 } & \text { I-HF5 } & \text { I-SS5 } & \text { I-HF5 } & \text { I-PA1 } \\ 2 & \text { V-PA1 } & \text { V-PA1 } & \text { I-HF5 } & \text { i }- \text {-PA1 } & \text { I-HF5 } & \text { I-PA1 } \\ 3 & \text { i-HF5 } & \text { i-PA1 } & \text { V-PH1 } & \text { i-PA1 } & \text { i-HF5 } & \\ 4 & \text { IV-PA1 } & \text { V-PA1 } & \text { V-PA1 } & \text { ii-HF5 } & \text { I-PA1 } & \\ 5 & \text { I-IA3 } & \text { I-PA1 } & \text { I-PA1 } & \text { V-PA1 } & \text { ii-HF5 } & \text { (etc.) } \\ 6 & \text { I-IA3 } & \text { I-IA3 } & \text { V-PA1 } & \text { I-PA1 } & & \\ 7 & \text { I-IA3 } & \text { I-PA1 } & \text { Vi-PA1 } & \text { V-PA1 } & \text { I-SS5 } & \text { (etC.) } \\ 8 & \text { i-PA1 } & \text { III-PA1 } & \text { i-PA1 } & \text { III-IA3 } & \text { iV-PA1 } & \text { (etC.) } \\ 9 & \text { V-PA1 } & \text { I-PA1 } & \text { ii-PA1 } & \text { Vi-PA1 } & \text { V-IA5 } & \text { I-PA1 } \\ 10 & \text { i-HF5 } & \text { i-HF1 } & \text { i-PA1 } & \text { VII-PA1 } & \text { i-HF1 } & \end{array}$

Fig. 11. Excerpt of tab-delimited text file with local key area and cadence type encodings. Column 1 lists the chorale number, and each successive column lists the encoded cadences in order of appearance.

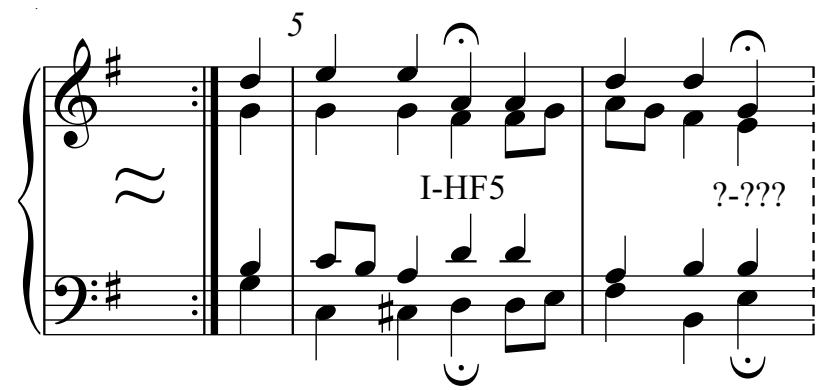

Fig. 12: The first two fermatas after the double bar in chorale 65

Other analytical issues involved the tonality of the chorale. In some cases, the global key of the chorale is not entirely clear. This situation was found to be fairly rare, though. Moreover, as I hope to show, the global key turns out to be less important than the local key implications in terms of what cadence type to expect given a particular melodic pattern. A potentially larger issue is the fact that some chorale melodies are not obviously tonal; instead, we might characterize them as modal. The work of Burns (1993, 1994, 1995), for instance, shows that chorales with modal melodies can be successfully analyzed by replacing characteristic tonal voice-leading paradigms with modal paradigms. Renwick (1997), however, argues that it is only the melody of a chorale, analyzed in isolation of the harmony as a single line, that is modal; otherwise, Bach sets the melody in a patently tonal harmonic context. (Note, for example, how Bach sets the Phrygian tune of "Herzlich thut mich verlangen" in both a minor context $[21,89,270,286$, 345] and a major context [74, 80, 98, 367].) Overall, the impact of modal melodies on this study is considered to be relatively low since the melodic encoding scheme captures any chromatic content. So whether, for example, the melodic fragment b7-6-5 is drawn from a Mixolydian melody, a Dorian melody, or a major (Ionian) melody with some chromaticism, this fragment represents a particular category of melodic structure that is tracked via the melodic encoding scheme described earlier.

One final concern is that some cadences are inherently ambiguous. The classic case of tonicization versus modulation comes to mind, where it may not be clear, for instance, whether we have a half cadence in the key of tonic or an authentic cadence in the key of the dominant. To explore this issue somewhat (i.e., the degree to which cadence analysis is a subjective task), I asked another Ph.D. in music theory (David Temperley) to analyze a portion of the chorales using the same encoding method described above. Within the test subset (about 10\% of the total), we had identical encodings for over $95 \%$ of the cadence events. It was found that our primary differences did, in fact, concern the issue of tonicization versus modulation. It 
will be worthwhile, therefore, to keep this issue in mind as the results are discussed. Nevertheless, while our agreement level fell short of the $100 \%$ ideal, it seemed good enough to indicate confidence that music scholars generally classify cadential events within the chorales in highly similar ways.

In total, the corpus is encoded with 2,124 unique fermata events. The frequency data reported in the following sections were calculated using custom computer scripts written in the Python programming language to parse the tab-delimited text files described above. Finally, it should be mentioned that all frequency data reported here ignore repeat signs; so, for example, chorale 1 is considered to have only six cadences total.

\section{SOME SPECIFIC RESULTS}

Before presenting raw frequency data from the study, it might be helpful to focus first on a few specific cases so as to make the results more tangible. For example, the beginning of this paper presented a melody in which the opening phrase ended with the scale-degree pattern 6-6-5 (Figure 1). There are 90 unique instances of this 6-6-5 phrase ending (measured in terms of the global tonic) within the chorales of Bach. How many of these instances employ a half cadence in tonic? (Figure 2 showed just one possible realization of this generic category.) The reader is urged to make an honest estimate; to encourage doing so, the answer has been moved to the endnotes.[9]

The result may be surprising, particularly given the grammatical validity of the counterpoint and harmonic progression in the hypothetical realization of Figure 2; a half cadence in tonic seems like an entirely reasonable choice for this melodic fragment. Gauldin did warn against cadences in the home key, though, so the result does appear to confirm that tonic-key tunnel vision leads to a poor approximation of the Bach style. But what of the various possible harmonizations shown in Table 1? Again, it would be worthwhile for the reader to consider their own estimates for each cadence category, some of which involve scale-degree reinterpretation (the last seven) while others do not (the first three). The results are shown below in Table 3.

Table 3. Distribution of cadence types given a melodic phrase ending 6-6-5.

\begin{tabular}{|c|c|c|}
\hline Cadence encoding & Sample Harmonization & Frequency \\
\hline I-PL5 & IV - IV - I & $8.9 \%$ \\
\hline I-HF1 & $\mathrm{IV}-\mathrm{ii}^{6}-\mathrm{V}$ & 0 \\
\hline I-IA5 & $I V^{6}-v^{107}{ }^{67}-I$ & 0 \\
\hline V-PA1 & $\mathrm{ii}^{6}-\mathrm{V}-\mathrm{I}$ & $83.3 \%$ \\
\hline V-DE3 & $i i^{6}-V-v i$ & $1.1 \%$ \\
\hline V-IA1 & ii $-V^{6}-I$ & 0 \\
\hline iii-IA3 & $i v^{6}-V^{7}-i$ & $4.4 \%$ \\
\hline iii-DE5 & $i v^{6}-V^{7}-V I$ & 0 \\
\hline iii-PL3 & $i v-i v^{6}-i$ & 0 \\
\hline IV-HF5 & $I^{6}-I V^{7}-V$ & $1.1 \%$ \\
\hline NC (No cadence) & N/A & $1.1 \%$ \\
\hline
\end{tabular}

As Table 3 shows, a perfect authentic cadence on the dominant is the clear frontrunner given a melodic phrase that ends 6-6-5. But while scale-degree reinterpretation is indeed necessary to achieve this cadence, other cadences involving scale-degree reinterpretation are much less common. Most notably, the second most common cadence given this melodic phrase ending - a plagal cadence in the home key - does not involve scale-degree reinterpretation. Based on these results (albeit for a single phrase ending), it appears that particular melodic patterns have particular cadential implications, which may or may not involve scale-degree reinterpretation.

This correspondence between melodic structure and cadence type turns out to be a prevalent feature of the chorales. In fact, tonic-key tunnel vision is not always a bad thing. One obvious situation occurs at the final cadence of a chorale. As many readers probably already know, the overwhelming majority of chorales end with a perfect authentic cadence in tonic. Indeed, 166 of the 177 major-key chorales end with a I-PA1; moreover, $99 \%$ end with a cadence in the tonic key. A similar situation can be found in the minor-key chorales. If we exclude those minor-key chorales with Phrygian melodies (all of which end on a dominant chord in relation to the home key, some via a half or Phrygian cadence, others via 
a modulation to the dominant), $99 \%$ of those that end on scale-degree 1 end also with a perfect authentic cadence on tonic.[10] Note that in minor-key chorales, final tonic cadences with Picardy thirds (I\#-PA1) outnumber those without a Picardy third (i-PA1) by a 10-to-1 ratio.

The value of tonic-key tunnel vision extends beyond just this somewhat trivial case of the final cadence. Consider, for instance, the melodic pattern 2-2-1. Since this phrase ending can occur in both major and minor keys, there are a number of cadential possibilities. But as shown in Table 4, even when the melodic pattern 2-2-1 occurs as a phrase ending other than at the end of the chorale, there is little evidence of scale-degree reinterpretation: over $97 \%$ of the 153 non-final fermata events given the melodic pattern 22-1 involve a cadence in the tonic key. Scale-degree reinterpretation thus seems like an appropriate technique in certain situations, less appropriate in others.

Table 4. Distribution of cadence types, excluding final cadences, given a melodic phrase ending 2-2-1.

\begin{tabular}{l|l}
\hline Cadence encoding & Frequency \\
\hline (I or i)-PA1 & $93.5 \%$ \\
(I or i)-DE3 & $3.9 \%$ \\
(I or i)-IA1 & 0 \\
(IV or iv)-PL5 & 0 \\
(IV or iv)-HF1 & 0 \\
(IV or iv)-IA5 & 0 \\
(vi or VI)-IA3 & $<1.0 \%$ \\
(vi or VI)-DE5 & 0 \\
(vi or VI)-PL3 & 0 \\
VII-HF5 & $<1.0 \%$ \\
NC (No cadence) & $1.3 \%$ \\
\hline
\end{tabular}

We could continue to investigate specific three-note melodic phrase endings and the events that most often associate with them, but there is not much utility (at least from a pedagogical standpoint) in providing a list of all three-note melodic phrase endings and the typical modulation schemes and cadence types for each. A broader approach is more profitable. In that vein, Table 5 shows cadence distributions in the major-key chorales given only the final scale degree at the cadential arrival. The most common and second-most common cadences are shown, along with the number of instances (\#) for each and the number of other event types. Note that final cadences have been excluded since (as discussed above) they are so predictable. Looking over this data, it seems clear that most cadence types fall into three categories: 1) a perfect authentic cadence (PA1);2) a half cadence with the chordal fifth in the soprano (HF5); and 3) an imperfect authentic cadence with the chordal third in the soprano (IA3).

Table 5. Distribution of cadences in the major-key chorales given the scale degree of the soprano at the cadential arrival, excluding final cadences.

\begin{tabular}{c|lr|lr|r|r}
\hline $\begin{array}{c}\text { Scale } \\
\text { Degree }\end{array}$ & Most common & & \multicolumn{2}{l|}{ Second-most common } & Others & \\
\cline { 2 - 5 } & cadence & $\#$ & cadence & $\#$ & $\#$ & Total \\
\hline $\mathbf{1}$ & I-PA1 & 156 & vi-IA3 & 35 & 57 & 248 \\
$\mathbf{2}$ & I-HF5 & 110 & ii-PA1 & 28 & 19 & 157 \\
$\mathbf{3}$ & I-IA3 & 100 & ii-HF5 & 36 & 27 & 163 \\
$\mathbf{4}$ & IV-PA1 & 16 & ii-IA3 & 10 & 10 & 36 \\
$\mathbf{5}$ & V-PA1 & 151 & I-PL5 & 12 & 49 & 212 \\
$\mathbf{6}$ & vi-PA1 & 21 & I-SS3 & 9 & 13 & 43 \\
$\mathbf{7}$ & V-IA3 & 36 & vi-HF5 & 17 & 8 & 61 \\
\hline Total & & 590 & & 147 & 183 & $\mathbf{9 2 0}$ \\
\hline
\end{tabular}

If we construct the same type of table for the minor-key chorales (shown in Table 6), we find a similar distribution of cadence types. Again, PA1, HF5, and IA3 cadences prevail. (Note that I have included both the raised and lowered versions of scale-degree 7 in this investigation, since both are commonly found at phrase endings in minor keys.) One notable exception to the hegemony of PA1, HF5, and IA3 cadences can be observed with the typical cadences for a raised scale-degree 7, which comprise 
half and Phrygian cadences in the tonic key. It is easy to explain this exception, however, since it is impossible to harmonize a raised scale-degree 7 in minor with any cadence in a closely-related (non-tonic) key area. (In other words, involving raised scale-degree 7 in a PA1, HF5, or IA3 cadence would require modulating to some key other than those that are closely-related.)

Table 6. Distribution of cadences in the minor-key chorales given the scale degree of the soprano at the cadential arrival, excluding final cadences.

\begin{tabular}{c|lr|lr|r|r}
\hline \multirow{2}{*}{$\begin{array}{c}\text { Scale } \\
\text { Degree }\end{array}$} & Most common & & \multicolumn{2}{|l|}{ Second-most common } & Others & \\
\cline { 2 - 5 } cadence & $\#$ & cadence & $\#$ & $\#$ & Total \\
\hline $\mathbf{1}$ & i-PA1 & 151 & i-DE3 & 15 & 31 & 197 \\
$\mathbf{2}$ & i-HF5 & 111 & VII-IA3 & 32 & 18 & 161 \\
$\mathbf{b 3}$ & III-PA1 & 110 & i-IA3 & 9 & 22 & 141 \\
$\mathbf{4}$ & iv-PA1 & 22 & III-HF5 & 20 & 7 & 49 \\
$\mathbf{5}$ & III-IA3 & 52 & v-PA1 & 47 & 101 & 200 \\
$\mathbf{b 6}$ & iv-IA3 & 6 & VI-PA1 & 2 & 1 & 9 \\
$\mathbf{b 7}$ & VII-PA1 & 37 & v-IA3 & 11 & 8 & 56 \\
$\mathbf{7}$ & i-HF3 & 28 & i-PH3 & 10 & 0 & 38 \\
\hline Total & & 517 & & 146 & 188 & $\mathbf{8 5 1}$ \\
\hline
\end{tabular}

Overall, the most-common and second-most-common cadences account for about $80 \%$ of the nonfinal cadential events in the Bach chorales. This figure is fairly high, and music theory students might benefit from memorizing all 30 of the cadence types listed in Tables 5 and 6 before their next chorale harmonization assignment. But 30 different cadence types is a lot for a student to remember, so some further refinement seems warranted.

\section{A MODEL FOR SCALE-DEGREE REINTERPRETATION}

Because PA1, HF5, and IA3 cadences are so prevalent in the distributions of Tables 5 and 6 , I would like to propose a simplified model of cadence types and local keys areas. The major-key version of this model is shown in Table 7. Given the scale-degree of the soprano at the cadential arrival (shown in the left-hand column), this table maps the resultant PA1, HF5, and IA3 cadences for closely-related key areas, with key areas organized from left to right by their typicality. (So given scale-degree 3 in the soprano at the fermata, for example, a first-level default would be an imperfect cadence in the tonic key, and a second-level default would be a half cadence in the key of the supertonic.)

Table 7. A simplified model for cadences and key areas in the major-key Bach chorales, given the scaledegree of the soprano at the cadential arrival.

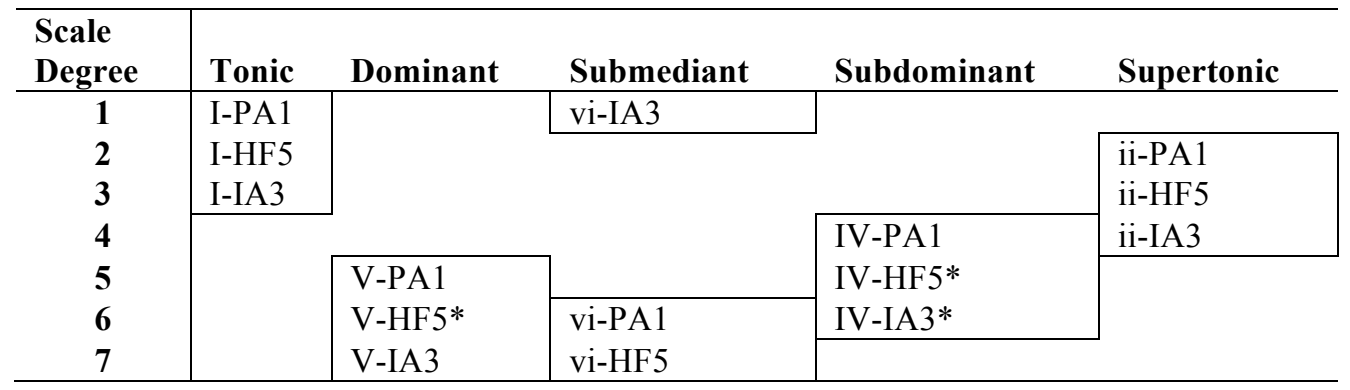

This simplified model would be easy for a student to remember: essentially, it proposes that a harmonization default is to interpret the soprano note at the fermata as scale degree 1 (via a perfect authentic cadence), 2 (via a half cadence), or 3 (via an imperfect authentic cadence) in tonic or some closely-related key area, with the tonic, dominant, and submediant keys being more likely destinations (in that order) than the subdominant or supertonic. So if a melodic phrase ends on scale-degree 7, for example, it would be more stylistic to modulate to the dominant (where scale-degree 7 would reinterpreted locally as 
scale-degree 3 ) or to the relative minor (where it would be reinterpreted locally as scale-degree 2) than to have a half cadence in tonic. Indeed, I-HF3 cadences are fairly rare in the major-key chorales, accounting for less than $10 \%$ of the cadences supporting scale-degree 7 . (This finding is a notable exception to the list of common cadences that Gauldin proposes shown in Figure 5.)

A minor-key version of this model can also be created, as shown in Table 8. The underlying strategy is the same as used to create Table 7, with PA1, HF5, and IA3 cadences mapped in terms of key areas organized from left to right by their typicality. Interestingly, while Table 6 shows that more cadences overall occur in the tonic key, it also shows that there is a tendency to modulate to the relative major whenever possible. For this reason, the mediant key (not the minor tonic) is the left-most key area in Table 8. We find evidence of this preference for the relative major elsewhere, as the subtonic (i.e., the dominant of the relative major) is a more probable key destination than the minor dominant.

Table 8. A simplified model for cadences and key areas in the minor-key Bach chorales, given the scaledegree of the soprano at the cadential arrival.

\begin{tabular}{|c|c|c|c|c|c|}
\hline $\begin{array}{l}\text { Scale } \\
\text { Degree }\end{array}$ & Mediant & Tonic & Subtonic & Dominant & Subdominant \\
\hline 1 & & \multirow{3}{*}{$\begin{array}{l}\text { i-PA1 } \\
\text { i-HF5 } \\
\text { i-IA3 }\end{array}$} & VII-HF5* & \multirow{7}{*}{$\begin{array}{l} \\
\\
\text { v-PA1 } \\
\text { (n/a) } \\
\text { v-IA3 }\end{array}$} & \multirow{6}{*}{$\begin{array}{l}\text { iv-PA1* } \\
\text { iv-HF5 } \\
\text { iv-IA3 }\end{array}$} \\
\hline 2 & & & VII-IA3 & & \\
\hline b3 & \multirow{3}{*}{$\begin{array}{l}\text { III-PA1 } \\
\text { III-HF5 } \\
\text { III-IA3 } \\
\end{array}$} & & & & \\
\hline 4 & & & & & \\
\hline 5 & & & & & \\
\hline b6 & & & & & \\
\hline b7 & & & VII-PA1 & & \\
\hline
\end{tabular}

Generally speaking, this simplified model approach does a decent job of accounting for cadence and modulation choices in the Bach chorales. Its success rate sits at $80.6 \%$ overall (1761 internal cadences, 1420 model matches): a good result, but not great. (The mismatches between the model and the data are noted with asterisks in Tables 7 and 8.) After closer analysis, it was found that the success rate of the model is closely linked to the melodic interval leading into the cadential arrival. For instance, most melodies descend by step into the cadential arrival (as shown in Table 9), and the model fares noticeably better in this situation, with a success rate of roughly $90 \%$. It is less successful at handling other intervallic patterns. For some of these melodic intervallic patterns - the ascending 3rd, descending 4th, and ascending 4th there are simply not enough instances in the Bach chorales to make any meaningful estimate of a typical solution. (Arguably, the case of unison melodic endings also suffers from too small a sample size.) For other intervallic patterns - the ascending 2 nd and descending 3 rd - some additional concepts seem to be required. Ideally, we would extend the model only slightly without sacrificing too much of its simplicity and pedagogical convenience.

Table 9. Success rate for the simplified model (PA1, HF5, IA3), excluding final cadences, given the interval leading into the cadential arrival.

\begin{tabular}{l|l|l|l}
\hline $\begin{array}{l}\text { Generic Melodic Interval } \\
\text { at Cadence }\end{array}$ & Instances & Model Matches & Success Rate \\
\hline Descending 2nd & 1084 & 968 & $89.3 \%$ \\
Ascending 2nd & 456 & 346 & $75.9 \%$ \\
Descending 3rd & 137 & 85 & $62.0 \%$ \\
Ascending 3rd & 1 &.. &. \\
Descending 4th & 14 & 8 &.. \\
Ascending 4th & 22 & 4 &. \\
Unison & 47 & 12 & $25.5 \%$ \\
\hline
\end{tabular}

After some trial and error, it was found that by adding just four special cases to the core cadences of PA1, HF5, and IA3, we can boost the overall success rate well above $90 \%$. These special cases are the deceptive cadence, the plagal cadence, the subdominant stop, and a specific contrapuntal cadence. 
Admittedly, many of these special cases should not be surprising (especially in light of the statistics shown in Tables 5 and 6). But the way in which these cadence types manifest within the Bach chorales is worthy of some discussion.

The first special case - that of the deceptive cadence - accounts for $2.5 \%$ of cadences in the chorales overall. Figure 13 shows a few voice-leading paradigms, given a chorale in $\mathrm{C}$ major. (The last cadence in Figure 13 thus represents a V-DE3.) Post-hoc analysis shows that deceptive cadences seem to be used primarily to add harmonic variety to adjacent melodic phrases that end on the same note. In particular, there is a significantly higher incidence of deceptive cadences as the penultimate cadence $(p<.01$; FET).[11]

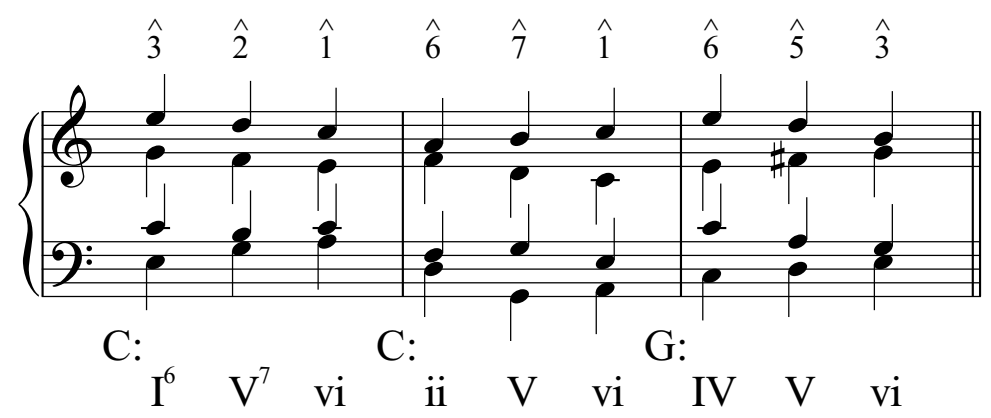

Fig. 13. Voice-leading examples for deceptive cadences.

The second special case is the plagal cadence, which accounts for $2.8 \%$ of cadences overall. Figure 14 shows voice-leading paradigms for two common scenarios. The most common is the PL5 type, which typically arises out of melodic upper neighbor motion around scale-degree 5. The handful of PL1 cases all arise from a melodic phrase ending that repeats the same note. It is worth pausing here to point out that - aside from the upper-neighbor-motion plagal cadence - these first two special cases retain the basic advice of the simplified model to conceptualize the melody note at the cadential arrival as scale-degree 1,2, or 3 in tonic or some closely-related key area.

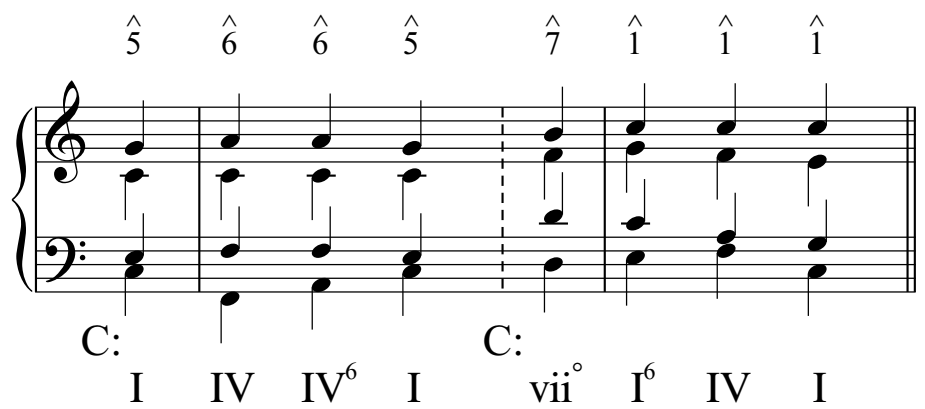

Fig. 14. Voice-leading examples for plagal cadences.

The third special case is the subdominant stop (described earlier), which accounts for $2.7 \%$ of all fermata events, i.e., roughly equal in frequency to both the deceptive and plagal cadences. Figure 15 shows two common voice-leading scenarios, both of which harmonize a descending melodic third from scaledegree 3 to 1 . Often, as in the second example of Figure 15, the local tonic is reinterpreted as $V^{7}$ of IV, which is interesting in that it creates a downwardly-resolving outer-voice leading tone. Overall, subdominant stops are especially more probable within the tonic key than in any other key $(p<.001$; FET).[12] 


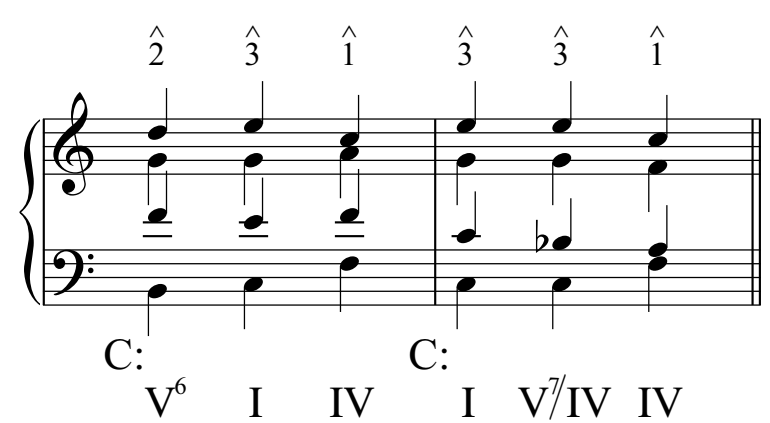

Fig. 15. Voice-leading examples for subdominant stops.

The final special case I refer to as "expansion to the octave." This case involves an ascending melodic line and a descending bass line, each moving by step into the final chord. It is worth noting that in every case of expansion to the octave, there is half-step motion in one of the outer voices at the cadential arrival. (So in a major key, for example, an expansion-to-the-octave half cadence will involve a raised scale-degree 4.) The benefit of this category is that it tidily encompasses a few different cadence types, including certain classes of imperfect authentic, half, and phrygian cadences, as shown in Figure 16.

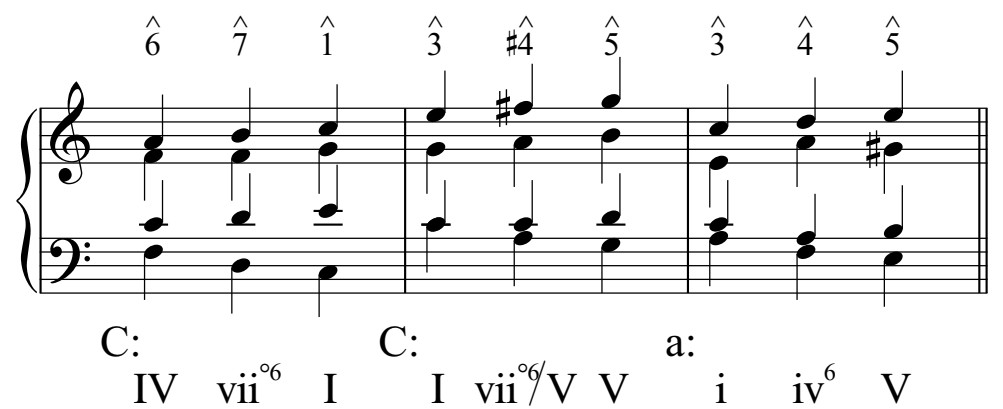

Fig. 16. Voice-leading examples for "expansion to the octave."

One could also refer to the expansion-to-the-octave cadence as a contrapuntal cadence (see Laitz 2012). Indeed, the examples in Figure 16 all fall into this larger category. But it is worth making a distinction between the specific scenario of stepwise outer-voice expansion and other flavors of the contrapuntal cadence, because the opposite scenario - in which the outer voices contract to an octave - is not a common feature in the Bach chorales. The exception is the "inverted" Phrygian cadence, shown as the first example of Figure 17, which ends many minor-key chorales. But while the other hypothetical voiceleading frameworks shown in Figure 17 seem like textbook examples of good harmony and counterpoint, they are not stylistically representative of the Bach chorale style.

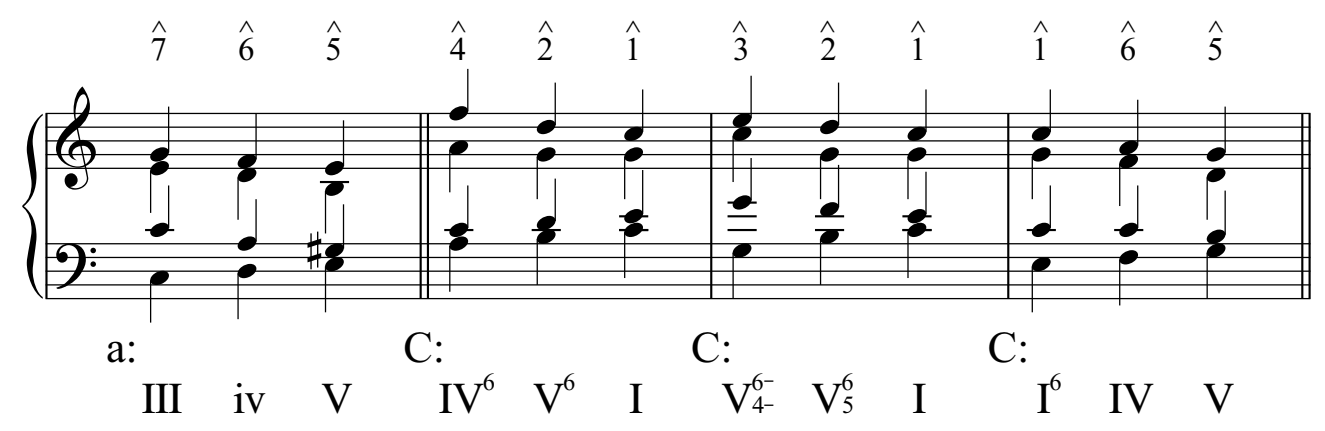

Fig. 17. Hypothetical voice-leading examples for "contraction to the octave."

As mentioned above, if we extend the simplified model of PA1, HF5, and IA3 cadences with these four special cases, our ability to account for cadences in the chorales is boosted well above the $90 \%$ mark. 
Specifically, Figure 18 organizes the trends described above into a flowchart of cadence choices that achieves a success rate of $92.2 \%$ in accounting for cadence choices in the chorales. Although this flowchart may look complicated, it represents a relatively straightforward conceptual approach to the scale-degree reinterpretation that Gauldin advises. Essentially, it suggests that the final note in the melody be interpreted as scale-degree 1, 2, or 3 in tonic or some closely-related key area, unless the final note is raised scaledegree 7 in minor (thus it must be a half cadence in tonic), part of an upper neighbor motion around scaledegree 5 (consider a plagal cadence in tonic), or ascended to by a whole step (consider a Phrygian cadence in tonic or some closely-related key).

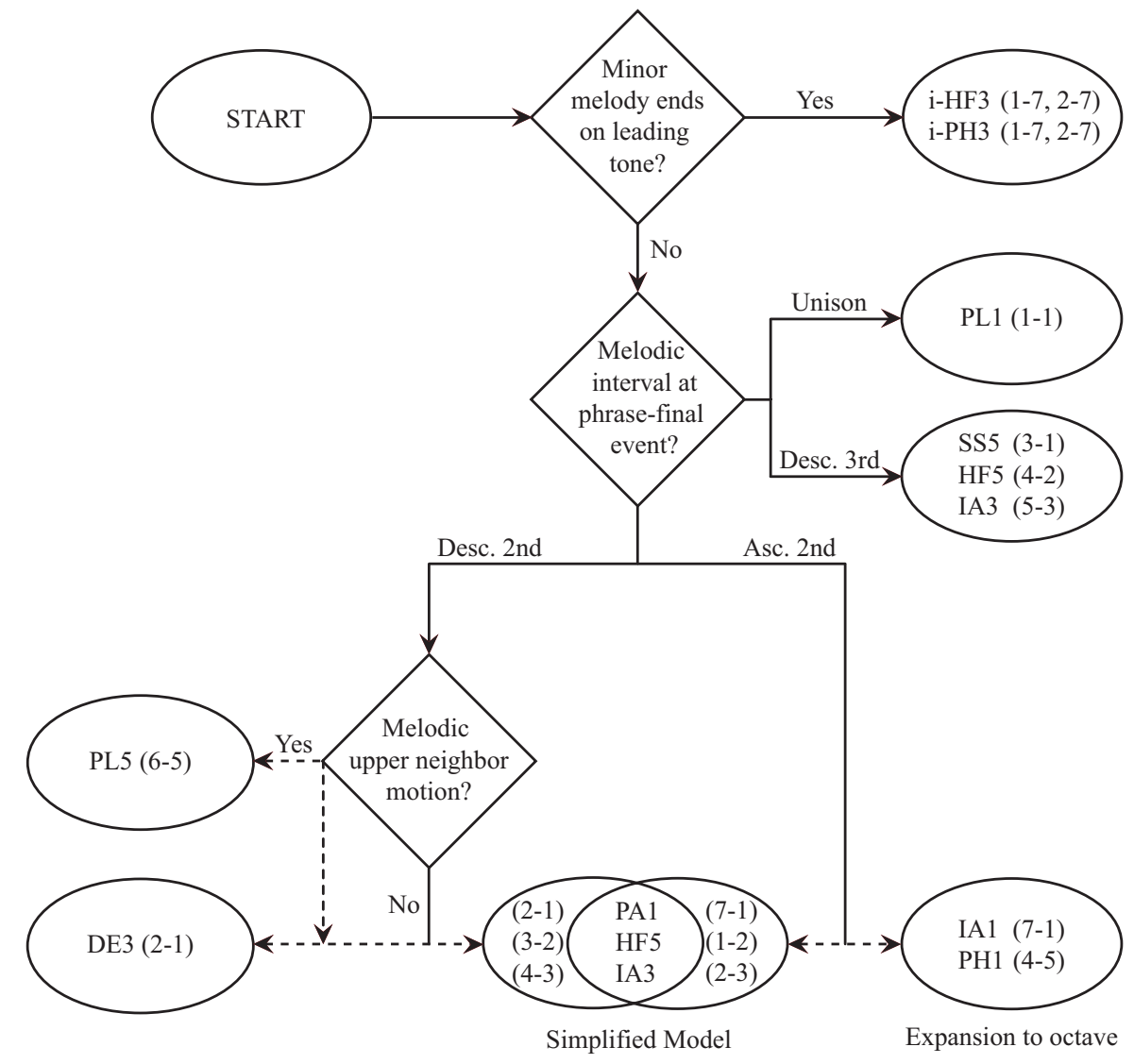

Fig. 18. Flowchart of cadence choices based on the melodic structure

\section{DISCUSSION}

In summary, we can say that a relatively small number of scenarios account for the vast majority of the $2,000+$ cadences in the chorale harmonizations of Bach. While a great amount of compositional variety can be found within these works, we observe a fairly consistent approach with regard to the way phrase endings are handled in terms of modulation schemes and cadence choices. Sometimes scale-degree reinterpretation is appropriate, sometimes it is not. With the general advice provided above, it will hopefully be easier to break students out of their "tonic-key tunnel vision," because it identifies what types of scale-degree reinterpretations are most typical while at the same time showing when this tonic-key tunnel vision leads to a stylistic solution.

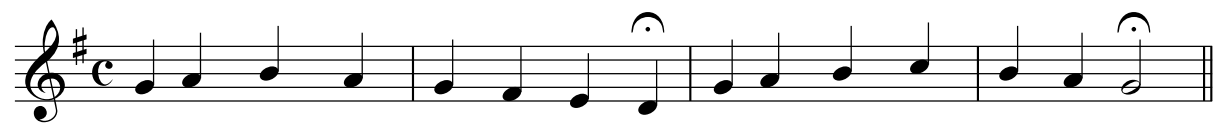

Fig. 19. Opening two phrases for the hymn melody "Freu' dich sehr, o meine Seele". 
As a final puzzle, consider the opening bars of the chorale melody "Freu' dich sehr, o meine Seele," shown in Figure 19 (in G major). The reader is encouraged to guess which cadence types Bach uses in his harmonization. After making an educated guess, consider the hypothetical harmonization of this hymn melody shown in Figure 20. How well does this realization jibe with the typical cadences and modulations discussed in the preceding paragraphs?

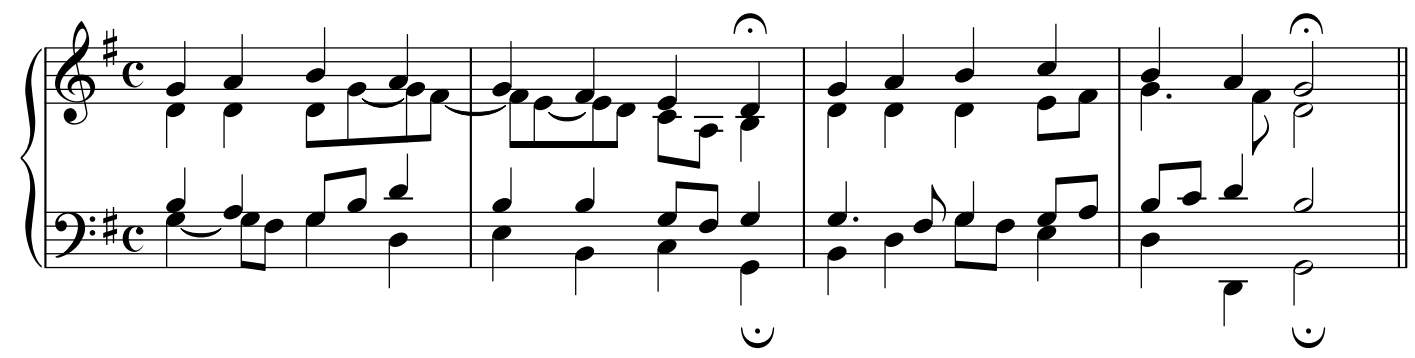

Fig. 20. Sample harmonization for the opening phrases of "Freu' dich sehr, o meine Seele" (Salzer and Schachter, 1969/1989, p. 294).

The harmonization in Figure 20 is not by Bach but is instead a sample harmonization found in the chapter on chorale harmonization from Salzer and Schachter's book, Counterpoint in Composition $(1969 / 1989$, p. 294). Of this example, the authors write that the first phrase - which could be categorized as ending with a plagal cadence in tonic - is "not bad" when considered in isolation (p. 295). In context with the second phrase, however - which also cadences in the tonic key - the first phrase proves to be "unsatisfactory," they contend, since "there is not enough variety, not enough relief from the constant emphasis on tonic harmony" (p. 295). Essentially, Salzer and Schachter use an explanation similar to Gauldin's advice to avoid tonic-key tunnel vision.

Salzer and Schachter support this explanation by comparing their hypothetical setting to that of Bach, shown below in Figure 21. Bach, as we can see, does not use consecutive cadences in tonic. Instead (as hopefully most readers estimated), Bach harmonizes the first phrase via a perfect authentic cadence in the dominant. Compared to Bach's harmonization, the harmonization by Salzer and Schachter is bound to seem inherently inferior. But we should take issue with the explanation that Salzer and Schachter provide as to why this is so. The first cadence in their harmonization is unstylistic, I would argue, not because it has two tonic cadences in a row; rather, the first cadence is unstylistic because of how it handles the melodic structure. It turns out that Salzer and Schachter's implicit advice to avoid consecutive tonic cadences is rather poor, as over a third of the chorales include consecutive tonic cadences. (Chorales 86 and 323 have five authentic cadences in tonic in a row!) Here again, we find evidence that tonic-key tunnel vision is not necessarily a bad thing; rather, the modulation strategy is dependent on the melodic structure.

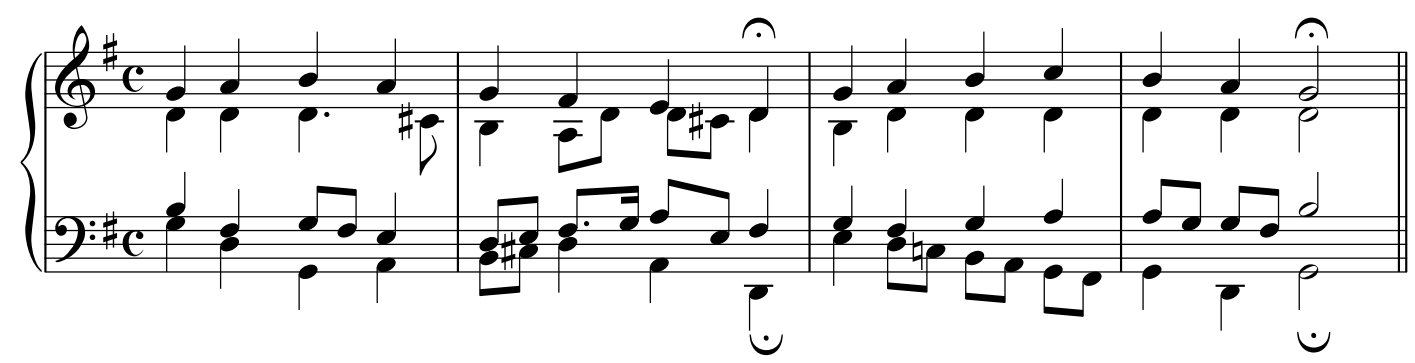

Fig. 21. Harmonization by J. S. Bach for the opening phrases of "Frue' dich sehr, o meine Seele," as found in chorale 64.

A few paradigmatic cases thus have great explanatory power. One might contend, however, that an emphasis on paradigms is not necessarily a good thing. Salzer and Schachter, for example, specifically warn that "nothing could be more stifling to musical development than to restrict the student to the most typical, frequently encountered usages" (1969/1989, p. 305). At issue is how much of what we teach is tied to universal truths, and how much is tied to a specific era, style, artist, or work. There is no clear answer to this question. In my own experience, I have found that the teaching of music theory is a constant balance 
between boundaries and freedom. A benefit of paradigms is that they act as solutions known to be good (or at least not bad) without introducing abstract prohibitions (against which students often react). Whatever one's personal belief, it would seem hard to argue that the knowledge of what is typical or frequently encountered is inherently something to the detriment of the student.

Similarly, some readers may feel that by focusing on melodic structure as a determinant of modulation and cadence choices, this study has ignored other important factors in Bach's compositional process. For example, it is entirely feasible that Bach wove into the musical fabric extra-musical considerations, such as the text of the hymns. The work of Pirro (1907/2010), for example, reveals a close relationship in the chorales between the music and its words. Undoubtedly, the structure of the melody goes only so far to explain every cadential choice, and it may be that some chords and progressions are only possible to reconcile through an analysis of the lyrics (or some other musical parameter). Nonetheless, this study has shown that we can account for a great deal of the cadential choices in the chorales via knowledge of the melodic structure alone. With the insights described herein, it may be that we can better identify and appreciate those strange and unique situations that are engendered by those factors not considered here.

One other outstanding issue is the extent to which Bach himself altered the melodies to suit his compositional goals. It was assumed in this study that the hymn tunes were pre-compositional entities, i.e., that Bach's modulation and cadence choices were tailored to fit the melody. But the actual scenario (at least in some cases) may be the reverse. It was noted earlier, for example, that Bach sometimes varies the hymn melody from one setting to another. Yet such variations are not common and presumably cannot greatly impact the fundamental melodic structure if the melody is to still be recognizable.

A number of possible factors could thus also be considered with regard to the small slice of the harmonization process that is cadence choice. Even with some hypothetically perfect knowledge of modulation schemes and cadence choices, moreover, we would presumably want to know more about many related aspects, such as the voice leading of the inner parts or the particular bass patterns that associate with each cadence type. Some cadence categories include information as to the latter, e.g., a perfect authentic cadence implies root-to-root motion in the lowest part; but others, such as the imperfect authentic cadence, do not. We saw with the notion of outer-voice expansion-to-the-octave that a single contrapuntal concept could account for various different cadence categories. It would be interesting in a future study, therefore, to investigate what types of bass patterns associate with particular melodic patterns. Studying cadential bass patterns would add a more objective element to the somewhat subjective task of cadence classification. The results of such a study may even suggest new or overlapping cadence categories beyond those considered here.

\section{NOTES}

[1] Correspondence concerning this article should be addressed to: Trevor de Clercq, Department of Recording Industry, 1301 East Main Street, Middle Tennessee State University, Murfreesboro, TN, 37130, USA, trevor.declercq@mtsu.edu.

[2] In this article, I employ standard American nomenclature for cadence categorization, as found in undergraduate music theory textbooks such as those by Kostka, Payne, \& Almén (2013), Clendinning \& Marvin (2010), and Laitz (2012).

[3] Riemenschneider (in Bach, 1941/1986, p. viii) reports that the first manuscript copies date from 1764.

[4] As of May 29, 2014, the Riemenschneider edition is the top search result for "Bach chorales" on amazon.com.

[5] According to Riemenschneider in his notes to the chorales (Bach, 1941/1986, p. 139), chorale 150 was harmonized by Johann Rosenmüller.

[6] Chorales 326 and 125 present a hybrid case, in that 326 duplicates 125 through the first three fermatas but then introduces new material prior to the last two fermatas. For the sake of this study, I take these two chorales to be unique harmonizations. 
[7] By this method, the following chorales are ignored in this study, formatted as "copy (original)": 88 (23), 126 (100), 150 (not Bach), 177 (148), 178 (53), 195 (86), 256 (64), 257 (93), 259 (91), 282 (254), 295 (236), 302 (199), 305 (86), 306 (201), 307 (198), 308 (156), 309 (5), 318 (144), 319 (235), 328 (131), 349 (120), 353 (313), 354 (248), 361 (9), 365 (233).

[8] The encoded tab-delimited text files used in this corpus study can be downloaded from: $<$ http://www.midside.com/publications/chorales/>.

[9] Of the 90 instances of the melodic phrase ending 6-6-5 in the chorales of J. S. Bach, none are harmonized with a half cadence in tonic.

[10] A handful of minor-key chorales end with a melodic note other than scale degree 1 at the final fermata. In such situations, the alternative to a PA1 is fairly obvious. For example, chorale 262 (in G minor) has as its final fermata the melodic note A, which supports a i-HF5.

[11] Specifically, Fisher's exact test shows that the proportion of deceptive cadences at penultimate cadences to deceptive cadences at other cadence locations is significantly higher than the proportion of other cadence types at penultimate cadences to other cadence types at other cadences locations.

[12] Specifically, Fisher's exact test shows that the proportion of subdominant stops in the tonic key to subdominant stops not in the tonic key is significantly higher than the proportion of other cadence types in the tonic key to other cadence types not in the tonic key.

\section{REFERENCES}

Allan, M. \& Williams, C. (2005). Harmonising chorales by probabilistic inference. In L. K. Saul, Y. Weiss, \& L. Bottou (Eds.), Advances in Neural Information Processing Systems 17 (pp. 25-32). Cambridge, MA: MIT Press.

Bach, J. S. (1986). 371 Harmonized chorales and 69 chorale melodies with figured bass. (A. Riemenschneider, Ed.). New York: G. Schirmer. (Original work published in 1941)

Biyikoglu, K. (2003). A Markov model for chorale harmonization. In R. Kopiez, A. C. Lehmann, I. Wolther, \& C. Wolf (Eds.), Proceedings of the 5th Triennial European Society for the Cognitive Sciences of Music Conference (pp. 81-84). Hannover: ESCOM.

Boyd, M. (1999). Bach: Chorale harmonization and instrumental counterpoint. London: Kahn \& Averill. (Original work published in 1967)

Burns, L. (1993). J. S. Bach's mixolydian chorale harmonizations. Music Theory Spectrum, 15(2), 144-172.

Burns, L. (1994). Modal identity and irregular endings in two chorale harmonizations by J. S. Bach. Journal of Music Theory, 38(1), 43-77.

Burns, L. (1995). Bach's modal chorales. Stuyvesant, NY: Pendragon Press.

Caplin, W. (2004). The classical cadence: conceptions and misconceptions. Journal of the American Musicological Society, 57(1), 51-118.

Clendinning, J. P. \& Marvin, E. W. (2010). The musician's guide to theory and analysis (2nd ed.). New York: W. W. Norton.

Ebcioğlu, K. (1988). An expert system for harmonizing four-part chorales. Computer Music Journal, 12 (3), 43-51. 
Ebcioğlu, K. (1990). An expert system for harmonizing chorales in the style of J. S. Bach. The Journal of Logic Programming, 8, 145-185.

Gauldin, R. (1995). A practical approach to eighteenth-century counterpoint. Long Grove, Illinois: Waveland Press. (Original work published in 1988)

Gauldin, R. (2009). The evolution of a styles simulation course for graduate theory students. Journal of Music Theory Pedagogy, 23, 101-121.

Kostka, S., Payne, D., \& Almén, B. (2013). Tonal harmony with an introduction to twentieth-century music (7th ed.). New York: McGraw-Hill.

Hörnel, D. \& Menzel, W. (1998). Learning musical structure and style with neural networks. Computer Music Journal, 22(4), 44-62.

Laitz, S. (2012). The complete musician (3rd ed.). Oxford: Oxford University Press.

McHose, A. (1947). The contrapuntal harmonic technique of the 18th century. New York: Meredith Publishing.

Pirro, A. (2010). L'esthétique de Jean-Sébastien Bach. Charleston, SC: Nabu Press. (Original work published in 1907)

Quinn, I. (2010). Are pitch-class profiles really "key for key"? Zeitschrift der Gesellschaft für Musiktheorie, 7(2), 151-163.

Quinn, I. \& Mavromatis, P. (2011). Voice-leading prototypes and harmonic function in two chorale corpora. In C. Agon, E. Amiot, M. Andreatta, G. Assayag, J. Bresson, \& J. Manderau (Eds.), Proceedings of the 3rd International Conference of the Society for Mathematics and Computation in Music (pp. 230240). Berlin: Springer.

Renwick, W. (1997). Bach and the "old” musicology. Music Analysis, 16 2), 255-269.

Rohrmeier, M. \& Cross, I. (2008). Statistical properties of tonal harmony in Bach's chorales. In K. Miyazaki, M. Adachi, Y. Hiraga, Y. Nakajima, \& M. Tsuzaki (Eds.), Proceedings of the 10th International Conference on Music Perception and Cognition, (pp. 619-627). Adelaide: Causal Productions.

Salzer, F. \& Schachter, C. (1989). Counterpoint in composition. New York: Columbia University Press. (Original work published in 1969)

Thorpe, C. (1998). C. P. U. Bach: using Markov models for chorale harmonization. Unpublished bachelor's thesis, Harvard College, USA.

Tymoczko, D. (2011). A geometry of music: harmony and counterpoint in the extended common practice. Oxford: Oxford University Press. 